\section{Verhaltensmedizinische Aspekte und Therapie des chronischen Tinnitus}

Gerhard Goebel

Medizinisch-Psychosomatische Klinik Roseneck, Prien am Chiemsee

psychoneuro 2004; 30 (6): 330-236

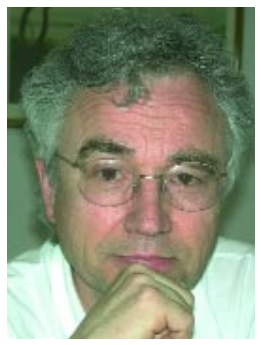

Gerhard Goebel
Wenn sich ein Tinnitusbetroffener in ärztliche Behandlung begibt, wird er zunächst gefragt, was ihm fehle. Er beschreibt dann sein Symptom, das konstant oder inkonstant wahrgenommen wird. Erst danach folgt die eigentliche Antwort: Es fehlt die Ruhe und Stille! Um das Ausmaß des Leidens in verständlichem Umfang zu verstehen, ist neben der HNO- und neurologischen Diagnostik u.U. auch eine Verhaltensanalyse mit Erfassung des Tinnitusschweregrades sowie der psychiatrischen Komorbidität zu erheben. So finden sich z. B. in neurootologischen Spezialambulanzen bei knapp zwei Drittel der Patienten gegenwärtig oder zurückliegend psychische Störungen. Ein verhaltenstherapeutischer Zugang, der auf der Basis systematischer Behandlungskonzepte eine Bewertungs- und Verhaltensänderung erreicht (kognitive Verhaltenstherapie), die psychiatrische Komorbidität und sozialmedizinischen Aspekte berücksichtigt und zu Maskierungsmöglichkeiten mittels frühzeitiger Hörgeräteanpassung bzw. Hörtraining/ Rauschgenerator motiviert, ist aus derzeitiger Sicht eine effektive Behandlung.

$\mathrm{D}$ ie Tatsache, dass wohl jeder Mensch das Phänomen Tinnitus kennt, macht es schwierig, die Häufigkeit des Vorkommens und das Ausmaß der Belästigung zu bestimmen. Wir wissen zwar, dass bei Erhebungen in Deutschland beim Hausarzt ca. $15 \%$ und beim HNO-Arzt ca. 25\% der Patienten Tinnitus angeben und jährlich bei ca. 340000 der erwachsenen Bundesbürgern der Tinnitus in eine chronische Form übergeht. Viel schwieriger ist es allerdings, zu eruieren, warum für viele der Tinnitus nicht störend ist und andere unter dem Dauergeräusch verzweifeln.

\section{Wer leidet unter Tinnitus? Bezug zu psychoakustischen Un- tersuchungen}

Patienten mit äquivalenten Tinnitusfrequenzen und Tinnitusintensitäten geben in der Regel ein breites Spektrum von Reaktionen auf den Tinnitus an. Entsprechend sind verschiedene Untersucher zu dem Ergebnis gekommen, dass unterschiedliche psychoakustische Parameter allenfalls gering gradig mit den im Zusammenhang mit dem Tinnitus angegebenen Beeinträchtigungen und der Tinnitusbelastung korrelieren. So fand sich in mehreren Studien, dass psychologische Therapieverfahren eine deutliche Abnahme der Tinnitusbelastung und der subjektiven Tinnituslautheit bewirkten, ohne dass dies mit einer relevanten Veränderung bei den erfassten psychoakustischen Lautheitsmaßen (Tinnitusanalyse) einherging (7). Lediglich die Maskierungspegel der minimalen Verdeckung (Minimal Masking-Level MML) und die Unbehaglichkeitsschwelle (UBS) scheinen zumindest für das Monitoring bei Masker-Therapien sinnvoll zu sein (16). Auch scheint ein tonaler Tinnitus störender bzw. intensiver empfunden zu werden als nichttonale Tinnitusfor- men (9): Von 1544 befragten Amerikanern skalierten diejenigen mit tonalem/klingelndem Tinnitus die Lautheit ihres Tinnitus auf einer numerischen 100-mm-Analogskala (NAS) durchschnittlich mit 75 an, Personen mit einem rauschenden bzw. nichttonalem Tinnitus stuften ihn dagegen durchschnittlich mit 55 ein.

\section{Bezug zu Umgebungs- geräuschen}

Tinnitus, gleich welcher Genese (Abb. 1), wird von den meisten Betroffenen abhängig von Umgebungsgeräuschen als störend empfunden: Nach der repräsentativen Studie der Deutschen Tinnitus-Liga (DTL, Wuppertal) von 1999 wird der chronische Tinnitus von $37 \%$ nur bei Stille wahrgenommen, d.h. tagsüber wird er überhaupt nicht beachtet. Bei weiteren $44 \%$ lässt sich der Tinnitus durch Umgebungsgeräusche überdecken: Diese Menschen können sich zumindest mit Musik, Windgeräuschen z.B. beim Radfahren oder Nutzung von Masker bzw. Hörgeräten etc. vom Tinnitus ablenken. Schwerbetroffen dürften diejenigen 17\% der chronischen Fälle sein, deren Tinnitus sich selbst durch starken Lärm nicht übertönen lässt. Hierzu gehören unter Umständen auch Betroffene mit Tinnitus auf einem ertaubten Ohr (seltene Fälle von Hörsturz, nach Schädelbasisfraktur, Operation am Hörnerv wegen Akustikusneurinom etc.), denen 
auf der betroffenen Seite die Möglichkeit der Überdeckung mit Umgebungsgeräuschen völlig fehlt.

\section{Bezug zu psychiatrischer Komorbidität}

Etwa 10 bis $20 \%$ der chronisch Betroffenen leiden erheblich unter ihrem Tinnitus (dekompensierter Tinnitus): Die Patienten klagen über Hoffnungslosigkeit und Ängste, der Schlaf ist massiv gestört, zusätzlich bestehen Gedächtnis- und Konzentrationsstörungen. Bei diesen ca. eine Million Erwachsenen in Deutschland finden sich in fast $90 \%$ der Fälle psychische Störungen wie Major Depression (bis 67\%), Dysthyme Störungen (bis 21\%), Angststörungen (bis $31 \%$ ) und somatoforme Störungen (bis 32\%). Demgegenüber unterscheiden sich Erwachsene mit kompensiertem Tinnitus nicht wesentlich von der Durchschnittsbevölkerung, die auch ohne Tinnitus unter Depressionen etc. leiden können. Depression oder somatoforme Störungsbilder (Hypochondrie, Somatisierung), die bereits vor dem Auftreten eines Tinnitus bestanden haben, können auch als Risikofaktor der Entwicklung eines stressbezogenen Tinnitus angesehen werden $(2,5,11)$.

\section{Tinnitus als Stressfolge}

(psychosomatische Aspekte)

Die Vermutung, dass Stress Tinnitus verursacht, genießt große Popularität: So widmete sich 2000 das Heft 15 des Stern der Thematik unter der Titelblatt-Überschrift: „Stress im Ohr" Auch Gesundheitsratgeber und Fachwörterbücher verbreiten die Hypothese des stressbedingten Tinnitus. Die Betroffenen erleben dies so: Ca. $25 \%$ aller Tinnitusbetroffenen in Deutschland, die in der repräsentativen Studie der DTL befragt wurden, geben Stress als Ursache für den eigenen Tinnitus an.

Entgegen dieser Popularität der Tinnitus-Stress-Genese gibt es kaum ausreichend methodisch angemessene wissenschaftliche Untersuchungen. Schmitt et al. (15) fanden bei einer Befragung von stationären Patienten unmittelbar nach einem akuten Tinnitus- und Hörsturzereignis im Vergleich zu anderen soziodemographisch parallelisierten HNO-

Patienten deutlich intensivere Lebensbelastungen und Alttagssorgen. Zudem unterschieden sie sich von der Kontrollgruppe in ihren habituellen Stressbewältigungsmechanismen, indem sie mehr negative (Resignation, Selbstbeschuldigung, Flucht, etc.), erstaunlicherweise jedoch auch mehr positive (Situationskontrolle, Schuldabwehr, Ersatzbefriedigung, etc.) angaben.

Demgegenüber fanden andere Autoren keine spezifischen Auffälligkeiten. Betroffene, die ihren Tinnitus gut bewältigen können („,high copers") unterscheiden sich zwar von Betroffenen, die unlösbare Probleme mit den Ohrgeräuschen angeben ("low copers") und entsprechend allgemein psychopathologisch auffällig sind, zeigen jedoch nach Kirsch et al. bezüglich zurückliegender kritischer Lebensereignisse vergleichbare Belastungen (siehe 9, 13). Ebenso fanden Schneider et al. (siehe 9,13) keinerlei auffälligere Stressverarbeitungsmerkmale bei 32 Patienten mit chronischen Ohrgeräuschen im Vergleich zu einer klinischen Kontrollgruppe.

Bei den von Schmitt et al. (15) untersuchten Tinnitusbetroffenen zeigte sich keine Verbindung mit der Stressbelastung direkt vor Entstehung des Tinnitus. Auch eine jüngste

\section{Tab. 1 Stufenschema in Anlehnung an ADANO (1998) zur ambulanten und stationären Behandlung des chronischen Tinnitus}

Tinnitusschweregrad nach TF-Gesamtscore

Schweregrad I (TF-Score: $0-30$ )

VAS Tinnitusunannehmlichkeit: $0-40$,

kaum Leidensdruck

Schweregrad II (TF-Score: $31-46$ )

VAS Tinnitusunannehmlichkeit: $40-60$,

kompensierter Tinnitus; Tinnitus leicht störend

Schweregrad III (TF-Score: 47-59)

VAS Tinnitusunannehmlichkeit: $60-80$,

dekompensierter Tinnitus; Tinnitus quälend, nicht

selten psychische Komorbidität

Schweregrad IV (TF-Score: 60-84)

VAS Tinnitusunannehmlichkeit: $80-100$, dekompensierter Tinnitus, extremer Leidensdruck, meist psychische Komorbidität

(TF-Score= Globaler Schweregrad der Tinnitusbelastung im Tinnitus-Fragebogen (TF); Range:0 bis 84; nach Goebel \& von Wedel 2001
Komorbidität: bevorzugt stationäre Therapie gefolgt von ambulante TRT über 1 bis 2 Jahre nach psychologischer Diagnostik, evtl. integrierte ambulante Psychotherapie primär multimodale stationäre Psychotherapie mit Tinnitusbewältigungstherapie, ggf. überleitend in ambulante Psychotherapie

\section{Goebel \& Hiller 1998; VAS= Visuelle Analogskalen; TRT=Tinnitus-Retraining-Therapie)}

Therapeutische Konsequenz ein- bis zweimaliges Counseling durch

Counseling, Relaxationsverfahren, TRT nach psychologischer Diagnostik

Abwägung: bei psychischer nach Coebel 8 von Wedel 2001
Untersuchung bei 48 Patienten mit einem erst kurz bestehenden Tinnitus ergab sich im Vergleich zu geden Kontrollpersonen keine unAnspannung/Nervostat oder Arbeitsbelastung (3). Die nen, deren Tinnitus nach eigenen Angaben erstmalig nach Stress oder seelischer Belastung aufgetreten sei, keine unterscheidbaren Belastungen nach den operationalisierten Frageoergleich zu den KontrollAus den

Aus den Ergebnissen der letzten stressbedingten Tinnitusentstehung in ihrer allgemeine Aussage angezweifelt werden!

\section{Tinnitus als Stressor (somato-} psychische Aspekte)

Bewertungsstile können zu kaastrophisierenden Reaktionen auf Gefühl "Stressor") mit dem leben kognitiver, emotionaler, motivationaler und behavioraler Defizite führen. So geben Tinnituspatienten, die ihren Tinnitus als erheblichen Stressor bezeichneten, vermehrt (5). Vor gedankliche Reaktionen der Betroffenen auf den Tinnitus gelten als maßgeblicher Einfluss auf die 
Empfindung, den Tinnitus als Stressor verantwortlich zu machen (9): So zeigte eine Untersuchung bei 50 schweizerischen Tinnitusbetroffenen, dass es Betroffenen mit bekannter Tinnitusursache besser geht als Betroffenen mit unklaren oder unbekannten Tinnitusursachen (,idiopathischer Tinnitus”). Letztere fühlen sich besorgt, weniger selbstsicher, weniger durchsetzungsfähig, weniger wert und wenig ausgeglichen. Schmitt et al (15) fanden in Ihrer prospektiven Untersuchung drei Monate nach dem Akutereignis eine signifikante aber mäßige Verbindung zwischen der Chronifizierung des Tinnitus und external-fatalistischer Kontrollüberzeugung bzgl. Krankheit und Gesundheit sowie den Negativstrategien der Stressbewältigung. Je schlechter die Befindlichkeit zum Zeitpunkt des Tinnitus- und Hörsturzereignisses war, umso wahrscheinlicher kam es zu einem Persistieren des Tinnitus (15).

Patienten, denen es nicht gelingt, ihre Aufmerksamkeit vom Tinnitus abzuwenden, leiden mehr unter der Problematik. Beispielhaft gilt dies für nächtliche Ruhezeiten, in denen durch Abnahme der externen Stimulierung und damit einhergehender erhöhter Wahrnehmungsfähigkeit des Hörsystems der Tinnitus als „lauter“ eingeschätzt wird und die damit einhergehende zunehmende Fokussierung mit Zunahme des
Belästigungsgrades korreliert. Newman et al. (siehe 5,9) fanden in einer sorgfältigen Analyse, dass dekompensierte chronische Tinnituspatienten mit hoher Selbstbeobachtung und körperlicher Empfindlichkeit durch hohe Tinnitusbelastung und Depressivität auffielen. Patienten mit niedrigem Pegel klagten dagegen eher weniger über Tinnitus und waren nicht depressiv. Diese Befunde bestätigen eigene empirische Untersuchungen, bei denen Patienten mit dekompensiertem Tinnitus durch eine hohe psychiatrische Komorbidität somatoformer und affektiver Störungen auffielen gegenüber Patienten mit kompensiertem Tinnitus, die keine somatoforme Störung bzw. eine psychiatrische Komorbidität entsprechend der durchschnittlichen Bevölkerung aufwiesen (5). In dieser Untersuchung konnte darüber hinaus belegt werden, dass Personen mit dekompensiertem Tinnitus neben dysfunktionalen Einstellungen zu Körper und Gesundheit im allgemeinen auch dysfunktionale Gedanken bezüglich des Symptoms Tinnitus haben: Sie bewerten sich körperlich schwach mit vegetativen Missempfindungen und neigen zu katastrophisierenden Bewertungen. Die Befunde weisen auf die moderierende Rolle von allgemeinen kognitiven Aspekten auf Verarbeitung und Wahrnehmungen der Tinnitusbelas-

\section{Abb. 1 Psychophysiologisches Tinnitusmodell $(11,13)$}

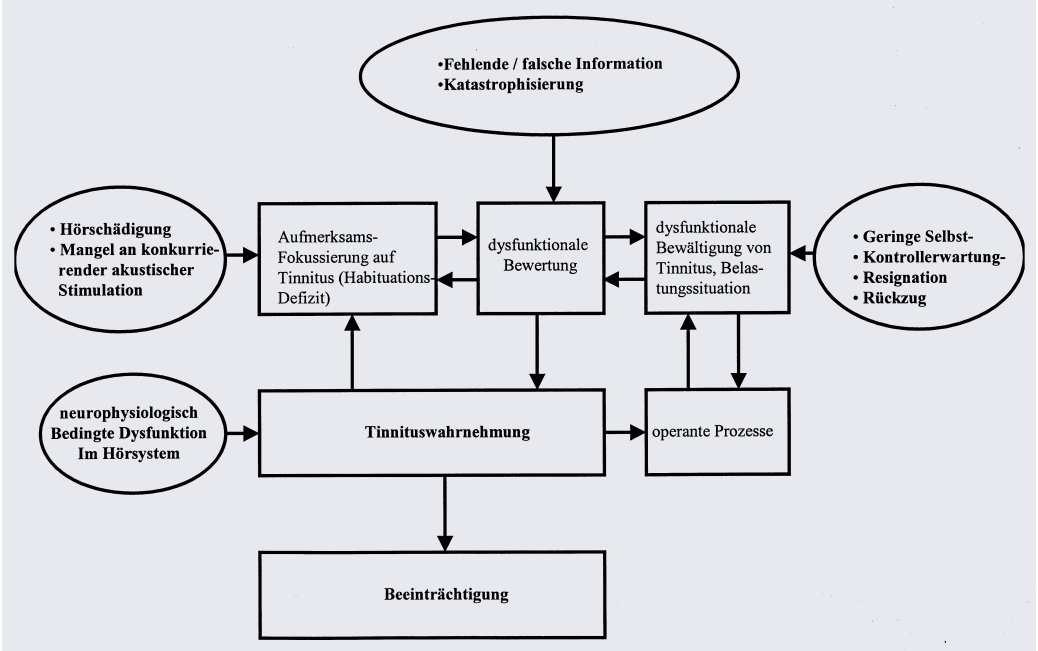

Somatische, psychische und soziale Faktoren sind an der Entstehung und Modulierung der Tinnitusbeeinträchtigung beteiligt

tung. Patienten mit dekompensiertem Tinnitus scheinen alltägliche Belastungen belastender als Patienten mit kompensiertem Tinnitus zu bewerten $(9,13)$.

\section{Soziales Netz}

Bei einem Vergleich von Tinnituspatienten mit ohrgesunden $\mathrm{Pa}$ tienten stießen Schneider et al. (5, 9, 13) auf den Befund, dass bei den untersuchten Patienten ihrer Tinnitussprechstunde weniger soziale Unterstützung und mehr soziale Belastung genannt wurden. Einschränkungen in den sozialen Rollenfunktionen bei depressiven Tinnituspatienten wurde in einer schwedischen Untersuchung auch von deren Ehepartner bestätigt (9). Gefken \& Kurt (2) berichteten ebenfalls von Beeinträchtigungen im Sozialbereich von Tinnitusbetroffenen: So litten diejenigen, die mit der angebotenen sozialen Unterstützung unzufrieden waren, am meisten unter ihrem Tinnitus. Dagegen korrelierte die Praxis eines ausgewogenen Lebensstils (z.B. sorgsamer mit sich umgehen) mit einer geringeren Tinnitusbelastung. Reich \& Johnson (in 12) fanden bei 144 Tinnituspatienten erhebliche soziale Anpassungsschwierigkeiten. Patienten mit dekompensiertem Tinnitus klagten ähnlich häufig über psychosoziale Belastungen wie Patienten mit somatoformen Störungen (5).

\section{Tinnitusbelastung und morphologische Verän- derungen an kortikalen Repräsentanzen}

Neueste Untersuchungen gehen davon aus, dass Tinnitus als Folge von Reorganisationsprozessen des zentralen auditorischen Systems verstanden werden kann (14). In der Regel werden diese Veränderungen durch periphere Hörschädigungen ausgelöst, können aber auch persistieren, selbst wenn sich die Funktionsstörungen der Cochlea weitest gehend rückgebildet haben.

Magnet-Enzephalographische Untersuchungen (MEG) bei Tinnitusbetroffenen aber auch psychophysikalische Studien bei Personen mit Hörverlust zeigen, dass dies mit entsprechender Verzerrung der tonoto- 
pen Karte und veränderten Diskriminationsschwellen in der Nähe der Hörsenke einhergeht (Abb. 2 und 3). Die Tinnitusbelastung, erfasst mit dem Tinnitus-Fragebogen (TF; 6), korreliert überraschend hoch mit diesen morphologischen Veränderungen ( $r=0,77$; Elbert, persönliche Mitteilungen, 14). Es scheint somit im Gegensatz zu den psychoakustischen Paramtern (s.o.) - erstmals gelungen, die Tinnitusbelastung in einem morphologischen Substrat abzubilden: Je weiter die kortikale Tinnitusrepräsentanz erscheint, um so mehr scheint der Betroffene unter dem Tinnitus zu leiden.

\section{Pragmatische Therapie beim chronischen Tinnitus}

Moderne psychophysiologische Modelle des chronischen Tinnitus gehen davon aus, dass dysfunktionale Aufmerksamkeitsprozesse und mangelnde Habituation wesentlich $\mathrm{zu}$ psychosomatischer Belastung und Chronifizierung beitragen. Je nach Beeinträchtigungsgrad ist daher in vielen Fällen eine umfangreiche Aufklärung („Tinnitus-Counseling“), gezielte Entspannungsübungen, die kombinierte Nutzung von Umweltgeräuschen oder apparative Module mit Counseling (Tinnitus-RetrainingTherapie (TRT), ambulante (kognitive) Verhaltenstherapie sowie stationäre Psychotherapie indiziert (Tab. 1).

\section{Direktives Counseling}

Tinnitusbetroffene sehen ihre emotionalen und verhaltensmäßigen Reaktionen häufig als direkte und einzige Konsequenz der organisch bedingten Tinnitusbelastung: „Erst wenn der Tinnitus weg geht, kann es mir wieder besser gehen“; „Wegen des Tinnitus kann ich nicht schlafen“ oder „... bin ich nicht mehr belastbar" sind typische Kognitionen, die einen entsprechenden Teufelskreis in Gang halten. Hier ist eine fundierte Informationsvermittlung über medizinische Grundlagen des Hörorgans, Konzeptionen zur Entstehung von chronischem Tinnitus sowie Beratung über die Möglichkeiten und Grenzen therapeutischer Maßnahmen angebracht.

Hat sich der Patient in der Akutphase selbst angewöhnt oder wurde er von Ärzten oder Angehörigen sogar dazu aufgefordert, immer wieder prüfend hinzuhören (Sensitivierung), ob der Tinnitus noch da ist, ob er sich verstärkt oder verändert hat (Lerntheorie), so muss er im chronischen Stadium motiviert werden, sich dieses Verhalten wieder „abzugewöhnen“ (Desensitivierung). Dieser Vorgang erfordert Zeit, Motivation beim Patienten, Überzeugungsarbeit durch die beteiligten Fachleute und oft eine geduldige Auseinandersetzung mit den Ängsten des

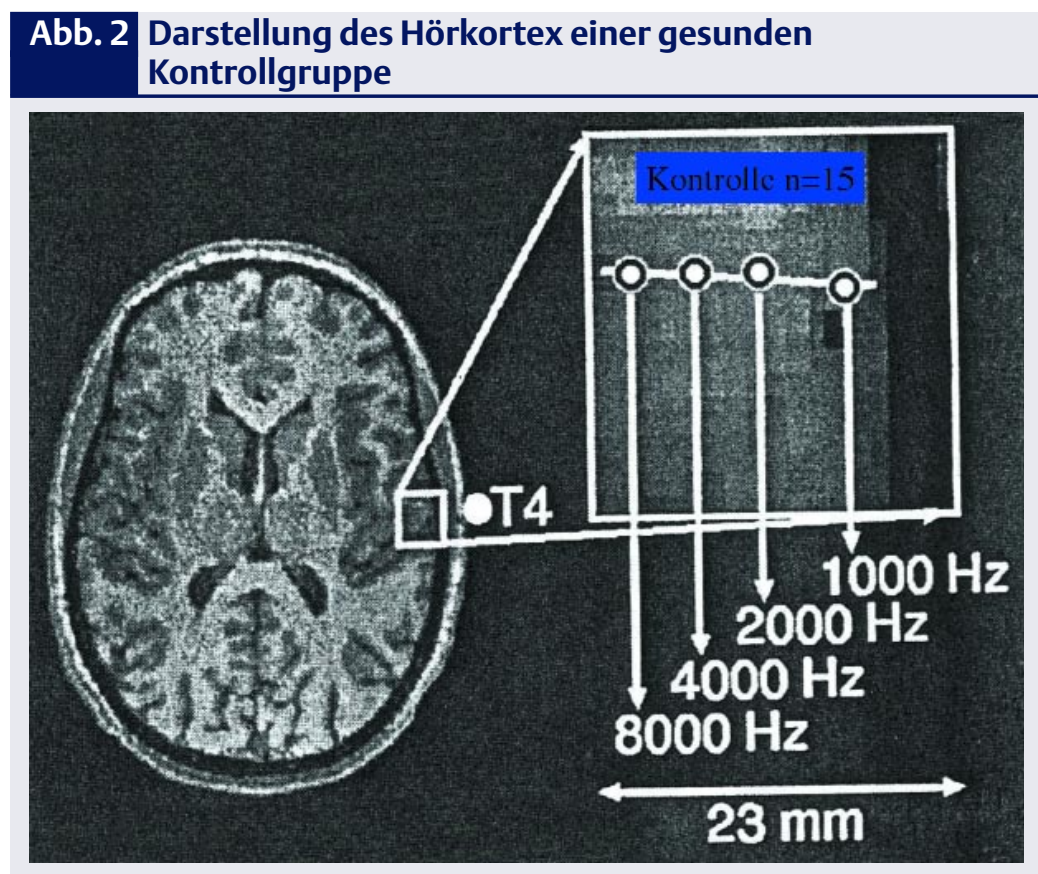

Man erkennt die mittels funktioneller Magnetenzephalographie abgebildeten Repräsentanzen der evozierten Frequenzen 1000 bis $8000 \mathrm{~Hz}$, die perlenschnurartig aufgereiht sind (nach 14)

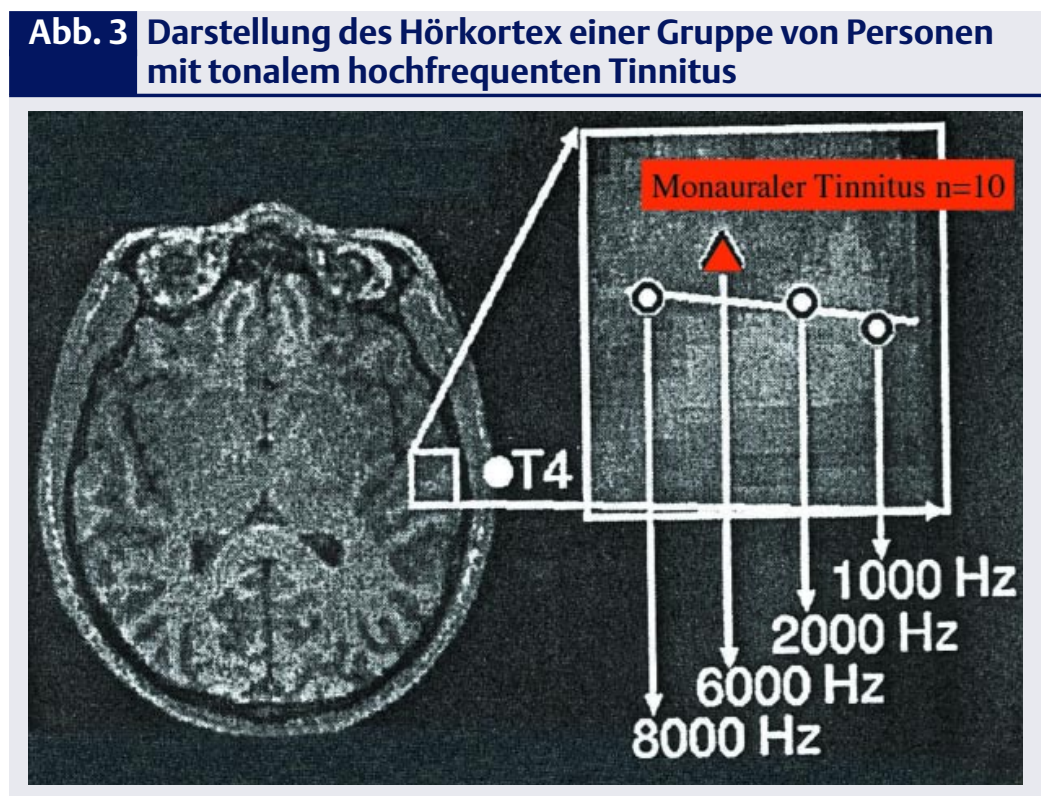

Man erkennt deutlich die Abweichung des simulierten Tinnitustones $(6000 \mathrm{~Hz})$ aus der tonotopen Linie (Pfeil) sowie ein „Auseinanderweichen“ der Nachbarfrequenzen $8000 \mathrm{~Hz}$ und $2000 \mathrm{~Hz}$ im Vergleich zu den gesunden Regionen der Kontrollgruppe (siehe Abb. 2) 
Patienten. Die Vorgehensweise lehnt sich hier an Therapiekonzepte medizinischer und verhaltenstherapeutischer Desensibilisierungsprogramme an. So, wie das Immunsystem langsam verlernt, auf ein Allergen zu reagieren oder ein Angstpatient durch die Therapie „verlernt“, beim Betreten eines Flugzeugs in Panik auszubrechen, sollen mit Hilfe des Counselings und besser noch durch kognitive Bewältigungsstrategien die Hörbahn und die mit ihr eng vernetzten Bereiche des autonomen und limbischen Systems „retrainiert" werden. Der Patient soll lernen, den bisher als Katastrophenalarm aufgefassten Tinnitus als ungefährliches und unwichtiges Signal zu interpretieren und schließlich zu überhören. Mittels Counseling und kognitiver Interventionen soll ein Abbau ungünstiger Befürchtungen eingeleitet werden und die Motivierung, auch nicht im direkten $\mathrm{Zu}$ sammenhang stehende psychische Beschwerden zu reduzieren, initiiert werden. Die behutsame Erweiterung

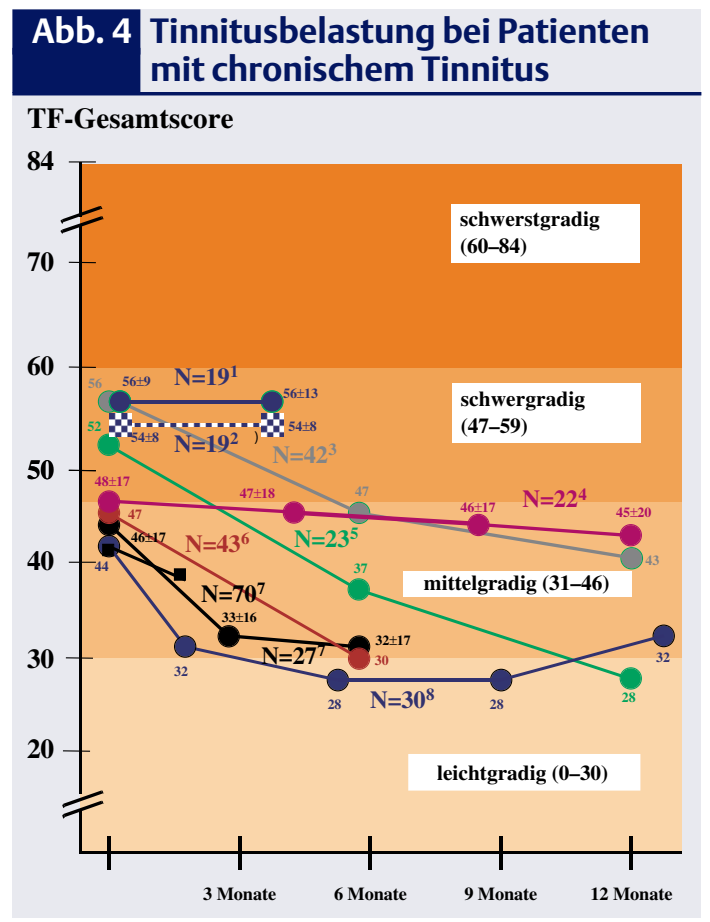

(1) Wartegruppe und (2) Rauschgenerator (RG)Gruppe ohne Counseling (7); (3) TRT mit RG: Biesinger et al., 1998 in 7; (4) Betreuung in HNO-Praxen: Goebel et al., 2003; (5) TRT mit RG bei Hyperakusis: Wölk und Seefeld, 1999 in 8; (6) TRT-Multizenterstudie: Bremer und Overhoff, 2001 in 8; (7) TRT + TBT: (2); (8) TRT nach Jastreboff: (13) des rein somatischen und eindimensionalen Tinnitusmodells hin zu einem psychosomatischen mehrdimensionalen Modell (Abb. 1) ist oft genügend für leicht bis mittelschwer Betroffene (Tab. 1). Alleiniges Counseling vermag je nach Schweregrad des Tinnitus in kurzer Zeit eine relevante Tinnitusgewöhnung $\mathrm{zu}$ initiieren $(8,13)$.

\section{Tinnitus Retraining-Therapie (TRT)}

Die Kombination des aktiven Counseling mit einer (passiven) apparativen Therapie (Hörgerät oder Masker, Geräuschtherapie) hat sich seit knapp 10 Jahren nach Vorschlag ihrer Protagonisten Jastreboff und Hazell auch im deutschsprachigen Raum unter der Bezeichnung TRT verbreitet. (8). Bei der TRT handelt es sich um ein Konzept und nicht um eine eigenständige Therapie. Sie orientiert sich an einem Tinnitusmodell, das Audiologen bzw. HNO-Ärzten, Psychotherapeuten und Hörgeräteakustikern gleichermaßen die Möglichkeit gibt, den Betroffenen die Vielschichtigkeit seines Tinnitus besser aufzuzeigen, als dies mit dem „Durchblutungsmodell“ oder dem „Stressmodell“ möglich ist. Tinnitus wird in diesem Modell als ein Problem der Wahrnehmung („Perzeption“) und weniger als ein Problem der peripheren Hörbahn („Stimulus“) verstanden (8).

Neu ist die Untermauerung dieses Modells durch die eingangs skizzierten Erkenntnisse, dass Tinnitus ähnlich dem Phantomschmerz - im Wesentlichen auch die Folge eines plastischen Prozesses zentraler Hörbahnbereiche ist (14).

Der Betroffene wird dabei motiviert, mittels eines relativ leisen Dauerrauschen eines Rauschgenerators (früher Masker) und/oder der Nutzung anderer Umgebungsgeräusche bzw. über die Verbesserung der Hörfähigkeit mit einem Hörgerät die Wahrnehmung des Tinnitussignal zu reduzieren, um damit die Habituation zu fördern $(8,9,13)$. Mit dieser „Hörtherapie“ soll die Wirkung des Counseling unterstützt werden, den Tinnitus zu akzeptieren. Die TRT-Interventionen erfolgen in etwa sechswöchigen individuellen Sit- zungen über Zeiträume bis zu zwei Jahren, in Deutschland auch in blockweisem Gruppendesign.

Auslassversuche bzw. vergleichende Evaluationen bis zu 18 Monaten zeigen, dass die Wirksamkeit des TRT überwiegend auf die kognitiven Therapieelemente des Counseling zurückzuführen sind $(3,13)$ (Abb. 4).

Tinnitusbewältigungs-Therapie (TBT), Tinnitus Retraining-Therapie (TRT) nach ADANO

Moderne psychophysiologische Modelle des chronischen Tinnitus gehen davon aus, dass dysfunktionale Aufmerksamkeitsprozesse und mangelnde Habituation wesentlich zu psychosomatischer Belastung und Chronifizierung beitragen. Kritische Analysen der TRT à la Jastreboff \& Hazell zeigen entgegen den anfänglichen euphorischen Erfolgsberichten eher moderate Wirkungen $(2,8,13)$. Um die Effizienz und Qualität zu steigern, werden die Patienten nach den Richtlinien maßgeblicher HNO-Fachgesellschaften im Team von HNOArzt und Psychotherapeuten/Psychologen ambulant behandelt. Kognitive Therapie in Kombination von Elementen der TRT (Counseling, Motivation zu Geräuschtherapie) steigert nachweislich positivere CopingFähigkeiten und Verbesserung des allgemeinen Erlebens und Verhaltens $(2,13)$. Bei gleichem Erfolg hinsichtlich der Minderung der Tinnitusbeeinträchtigung ist das TBT als Gruppendesign kostengünstiger und damit effizienter.

Oft ist es im Einzelfall schwierig zu entscheiden, ob die Bewältigungsanstrengungen mehr auf die Tinnitusproblematik zu konzentrieren sind (TBT), ob die somatischen Probleme wie Hörminderung und/ oder Geräuschempfindlichkeit (Hyperakusis) besonders zu berücksichtigen sind oder mehr die psychischen Störungen im Mittelpunkt zu stehen haben (z.B Angst- oder Depressions-Bewältigungstherapie). Hauptziel der kognitiven Therapie ist die Reduktion der endogenen negativen Tinnituswahrnehmung durch Modifizierung einer durch Verhaltensänderung zu erarbeitenden positiven Alternative (Refraim- 
ing). Dadurch wird es zunehmend ermöglicht, den Tinnitus zu ignorieren und schließlich zu „vergessen“ (9).

Biofeedback, Relaxation, Ablenkungstraining, Hypnotherapie

Einzelmethoden wie Biofeedback, Progressive Muskelentspannung (PME), Autogenes Training (AT) und Yoga zeigen als Einzelverfahren nur geringe Effekte. Integriert in eine Verhaltenstherapie kann Biofeedback den Relaxationsteil des Behandlungssettings unterstützen (in 7).

Die Wirkung von Biofeedback und Entspannungstraining kann neben der Beeinflussung des Vegetativum auch über Mechanismen der Defokussierung erklärt werden: Konzentriert sich der Patient während der Sitzungen auf die Instruktionen und den körperlichen Entspannungszustand, wird er allein dadurch die Ohrgeräusche vorübergehend weniger beachten: Es wird sich die Überzeugung einstellen, dass der Tinnitus kontrollierbar ist und das Gefühl der Hilflosigkeit wird abnehmen. Die damit erreichbare emotionale Ausgeglichenheit und Abnahme der ängstlich-phobischen Reaktion wird dazu beitragen, dem Tinnitus gegenüber gelassener zu werden, was die subjektive Lautheit reduzieren kann. Gerhards (in 13) konnten mit einem speziellen apparativ gestützten computergesteuerten Ablenkungs- und Entspannungstraining (AET) eine trainingsbedingte Verbesserung des Tinnitus-Ignorieren-Könnens und eine Reduktion der Belastung durch unterschiedliche psychische Symptome erreichen.

Die Literatur über den Einsatz von Hypnose bzw. Hypnotherapie in der Tinnitusbehandlung ist äußerst spärlich (7). Bei der Hypnotherapie wird kognitive Therapie mit Hypnose kombiniert. Im tiefen entspannten hypnotischen Zustand (Trance) wird der Patient instruiert, z. B. den Tinnituston in ein mehr akzeptierbares visuell-akustisches Bild zu verwandeln oder dem Tinnitus eine andere Bedeutung für sein Leben zu geben. Tonbandaufzeichnung der individuellen Sitzung ermöglicht

Tab. 2 Beispiel eines Behandlungsplans mit Therapieziel im Rahmen einer mehrdimensionalen Psychotherapie bei dekompensiertem Tinnitus

- Information über Tinnitusursachen (Tinnitusmodell; Counseling)

- Zusammenhänge mit Gedanken und Emotionen (kognitive Therapie)

- Aufgeben des Harmoniebedürfnisses (Psychotherapie)

- Eruierung eigener Bedürfnisse und Wünsche (Gruppenpsychotherapie)

- Aufbau von Durchsetzungsfähigkeit (Gruppentherapie soziale Kompetenz)

- Aufbau von Entspannungsfähigkeit (Entspannungsverfahren)

- Erlernen von angemessenem Verhalten im Alltag (Stressbewältigung)

- Exposition von Umgebungsgeräuschen und Stille (Hör-, Musiktherapie)

- Apparative Therapie (Hörgeräteakustiker)

dem Betroffenen zu Hause die Wirkung der Entspannung zu vertiefen. Korrekt durchgeführte Studien zur Untersuchung der Effizienz der Hypnotherapie fehlen im deutschen Sprachraum (7).

\section{Stationäre Psychotherapie \\ bei Tinnitus}

Besonders bei einem schweren bis sehr schwerst gradigen Leidensausmaß (Tab. 1) ist ein multimodaler stationärer verhaltensmedizinischer Therapieansatz indiziert (Tab. 2) und in seiner Effizienz belegt $(7,13)$. Bei 90\% dieser Patienten findet sich bei sorgfältiger Exploration ein oder mehrere psychiatrische Störungen, die in die Behandlung einbezogen werden müssen (5). In diesen Fällen ist primär oft nur das Mittel der stationären Krankenhausbehandlung prognostisch günstig ( $\$ 39$ SGB V). Entsprechend ist das Attest eines Facharzt für Psychotherapeutische Medizin oder Facharzt für Psychiatrie \& Psychotherapie zur Vorlage beim MDK der GKV bzw. Gesellschaftsarzt der PKV erforderlich.

In einer aktuellen Katamnesestudie werten wir die Daten von 500 konsekutiven Patienten mit chronisch dekompensiertem Tinnitus aus, die 1988 bis 1992 über einen Zeitraum von durchschnittlich sechs bis neun Wochen stationär mit Schwerpunkt kognitiver Verhaltenstherapie behandelt wurden. Im Vergleich zu einer Kontrollgruppe zeigte sich bei den behandelten Patienten klinisch relevante Verbesserungen im Tinnitus-Fragebogen (TF; Abb. $4 ;$ 6). Parallel zu den tinnitusbezogenen Beschwerden wurden die Verbesserungen auch in den Ta- gebuchprotokollen der Patienten sowie in den psychologischen Beschwerdemustern der SymptomCheck-List (SCL-90-R) deutlich. Etwa $20 \%$ der damals chronisch Betroffenen sind weitgehend remittiert, bei einem weiteren Drittel lässt sich der Tinnitus nur in ruhiger Umgebung wahrnehmen. Nach eins, zwei und zwölf Jahren zeigte sich insgesamt eine gute Stabilität der erreichten Therapieerfolge. Auch andere Katamnesestudien bestätigen für 75\% der meist schwerst Betroffenen die Effektivität einer stationären Verhaltenstherapie über sechs Monate (10). Als relevantes Therapierisiko werden ein offenes Rentenverfahren sowie ein fixiertes rein somatisch ausgerichtetes Krankheitsmodell, wie es bei ausgeprägten hypochondrischen Ängsten vorliegt, angesehen (12).

\section{Versorgung in Deutschland}

Die geschilderten therapeutischen Ansätze und Befunde machen deutlich, dass in Deutschland ein zunehmend differenziertes Repertoire von Behandlungsmethoden unter Einbeziehung medizinischer, apparativer und psychotherapeutischer Methoden vorgehalten werden kann. Mit entsprechender Gewichtung kann dieses Angebot in den unterschiedlichen Einrichtungen des Gesundheitssystems eingesetzt werden. Der Patient kann sowohl in der ambulanten HNO-ärztlichen Praxis, im Allgemeinkrankenhaus, bei verhaltensmedizinisch ausgerichteten ambulanten Therapeuten sowie in Fachkliniken mit einem auf Tinnitus bezogenen Behandlungsprogramm Hilfe finden. Es wird wei- 
ter erforderlich sein, Therapeuten zur speziellen Tinnitustherapie auszubilden, das Angebot der einzelnen Einrichtungen genauer aufeinander abzustimmen, klare Indikationskriterien für die eine oder andere Therapieformen festzulegen und der Chronifizierung durch unwirksame oder nicht ausreichende Behandlungen entgegenzuwirken.

\section{Fazit}

Die meisten chronisch Tinnitusbetroffenen benötigen keine intensive Behandlung mit psychotherapeutischem Schwerpunkt. In vielen Fällen ist eine umfassende Aufklärung über die Art der Erkrankung und mögliche Komplikationen ausreichend (Counseling), in anderen Fällen kann ein zusätzlich durch apparative Maßnahmen unterstütztes Habituationstraining die Tinnitusbelastung günstig beeinflussen. Für viele Patienten ist die Mitarbeit in einer Tinnitus-Selbsthilfegruppe wertvoll.

Beim dekompensierten Tinnitus und psychiatrischer Komorbidität sind stationäre Behandlungskonzepte zu bevorzugen*, die mit verschiedenen Behandlungskomponenten hier die besten Wirkungen erzielen. Der Patient soll einen $\mathrm{Zu}-$ stand erreichen, in dem er sich des Tinnitus nicht bewusst ist, obgleich er ihn unverändert hört, wenn er sich darauf konzentriert. Damit ist der Begriff „Heilung“ im Sinne der Abwesenheit von Tinnitus relativiert und gleichzeitig der mögliche Weg einer Tinnitusbewältigung aufgezeigt, der im letzten Stadium bei einem Drittel der Fälle in das komplette Sistieren des Tinnitus übergeht (4).

Wir müssen den betroffenen Patienten heute nicht mehr mit dem Satz abspeisen: „Damit müssen Sie leben“. Heute können wir ihm effektive Strategien aufzeigen, wie er mit seinem Tinnitus besser fertig wer-

\footnotetext{
*Die Selbsthilfeorganisation Deutsche Tinnitus-Liga (DTL) bietet Ärzten und Patienten einen Klinikwegweiser für Tinnitus, Hörsturz und Menière-Behandlung (Gebühr $15,-€)$ sowie eine Liste von ambulanten Tinnitus-Retraining-Therapie(TRT)-Zentren. www.tinnitus-liga.de/klinik-wegweiser.htm oder bei: Deutsche Tinnitus-Liga, Postfach 21035, 42353 Wuppertal
}

den und leben kann. Ziel ist dabei nicht zwingend die Beseitigung des Ohrgeräusches, sondern dass der Betroffene nicht mehr unter seinem Ohrgeräusch leiden muss. Dieses Ziel ist heute weitgehend erreichbar.

When someone with tinnitus seeks medical help, he is first asked what his problem is. He first describes his symptom and then gives the real answer: He lacks peace and quiet! In order to fully understand the scope of the problem, it is necessary to enhance the ENT and neurological diagnoses with a behavioral analysis, including the degree of tinnitus and psychiatric comorbidity. In outpatient treatment centers for otology about two thirds of the patients have current or past psychiatric disorders. From our view, a behavioral therapeutic approach with systematic treatment concepts, taking psychiatric comorbidity and social medical aspects into consideration and motivating patients to use masking and hearing aids or hearing training is an effective treatment.

\section{Literatur}

1. Delb W, D’Amelio R, Boisten CJM, Plinker PK. Kombinierte Anwendung von Tinnitusretrainingtherapie (TRT) und Gruppentherapie. HNO 2002; 50: 997-1004

2. Gefken R, Kurth H. Psychiatric problems due to tinnitus: results of a survey of patients with chronic tinnitus. In: Goebel,G. (Ed.) Psychosomatic Aspects of Complex Chronic Tinnitus. London, Quintessence publishing Co Surrey, 1998: 51-62

3. Gerhards F, Schwerdfeger FP, Etzkorn M, Hasselmeyer A. Psychosozialer Stress: Ein bedeutsamer ätiologischer Faktor bei Tinnitus? In: Dohrenbusch R \& Kaspers F (Hrsg.). Fortschritte der klinischen Psychologie und Verhaltenstherapie. Lengerich, Pabst, 2001:122-135

4. Goebel G, Büttner U. Grundlagen zu Tinnitus: Diagnostik und Therapie. Psychoneuro 2004; 30: 322-327

5. Goebel G, Fichter M. Depression beim chronischen Tinnitus. Münchner Medizinische Wochenschrift MMW 1998; 41: 557562

6. Goebel G, Hiller W. Tinnitus-Fragebogen (TF). Ein Instrument zur Erfassung von Belastung und Schweregrad bei Tinnitus (Manual). Göttingen, Hogrefe-Verlag: 1998 7. Goebel G. Wirksamkeit psychotherapeutischer Verfahren bei Tinnitus. In: Goebel G. (Hrsg.). Ohrgeräusche. Psychosomatische Aspekte des chronischen Tinnitus. München, Urban \& Vogel-Verlag, 2001: 97-124

8. Goebel G, von Wedel H. Tinnitus Retrai-
ning-Therapie. In: Goebel G. (Hrsg.). Ohrgeräusche. Psychosomatische Aspekte des chronischen Tinnitus. München, Urban \& Vogel-Verlag, 2001: 83-96

9. Goebel G. Tinnitus und Hyperakusis. In: Schulte D, Grawe K, Hahlweg K, Vaitl D. (Hrsg.). Fortschritte der Psychotherapie. Göttingen, Hogrefe-Verlag, 2003

10. Hesse G. Rienhoff N K, Nelting M, Laubert A. Ergebnisse stationärer Therapie bei Patienten mit chronisch komplexen Tinnitus. Laryngo-Rhino-Otol. 2001; 80: 503-508

11. Hiller W, Goebel G. Hals-Nasen-OhrenErkrankungen (HNO). In: Flor H, Hahlweg K, Birbaumer N (Hrsg.). Anwendungen der Verhaltensmedizin. Enzyklopädie der Psychologie: Klinische Psychologie, Vol 4. Göttingen, Hogrefe, 2001: 146-200

12. Hiller W, Goebel G. Assessing audiological, pathological and psychological variables in chronic tinnitus: A study of reliabiltiy and search for prognostic factors. International Journal of Behavioral Medicine 2000; 6: 312-330

13. Kröner-Herwig B. Tinnitus. In: Ehlert U. (Hrsg.). Verhaltensmedizin, Berlin, Heidelberg, Springer, 2003: 535-570

14. Pantev C, Weisz N, Schulte M, Elbert T. Plasticity of the Human Auditory Cortex. In: Pessoa P \& De Weerd P. (eds.) Filling-in: From Perceptual Comletion to Cortical Reorganisation. Oxford Univ. Press 2003, 231-251

15. Schmitt C, Patak M, Kröner-Herwig B. Stress and the onset of sudden hearing loss and tinnitus. International Tinnitus Journal 2000; 1: 41-49

16. von Wedel H. Apparativ-akustische Therapie bei Tinnitus. In: Goebel G. (Hrsg.): Ohrgeräusche. Psychosomatische Aspekte des chronischen Tinnitus. München, Urban \& Vogel-Verlag, 2001: 69-82

Korrespondenzadresse:

PD Dr. med. habil. Gerhard Goebel Chefarzt

Medizisch-Psychosomatische Klinik Roseneck

83209 Prien am Chiemsee

E-Mail: ggoebel@schoen-kliniken.de 